\title{
Recent Advances in Anaesthesia and Intensive Care-Volume 24
}

\author{
Jeremy N. Cashman, R. Michael Grounds. Cambridge University Press, 2007, 250 pp., \\ ISBN 978-0-521-70649-0
}

\author{
Derek R. Townsend, MD
}

Published online: 11 December 2008

(C) Canadian Anesthesiologists' Society 2008

First published in 1932 as Recent Advances in Anaesthesia and Analgesia (Including Oxygen Therapy), the book's title has changed over the years culminating in this 24th edition, Recent Advances in Anaesthesia and Intensive Care. This well organized 250-page text includes topics of interest to both general and specialist anesthesia practitioners, as well as to practitioners with an interest in critical care.

The following topics are covered in the book's 13 chapters: the role of cardiopulmonary exercise testing in the preoperative evaluation of surgical patients, esophagectomy for cancer, vascular surgery, anesthesia for the elderly, deaths following anesthesia, pelvic and acetabular trauma, echocardiography, levosimendan, critical care outreach, critical care and biological disasters, evaluating clinical performance, non-technical skills and anesthesia, and simulators in anesthetic training to enhance patient safety.

With its focus on up-to-date information and important concepts, the chapter regarding the role of cardiopulmonary exercise testing in the preoperative evaluation of surgical patients presents an excellent overview of the method to assess a patient with limited cardiopulmonary physiological reserve who requires major surgery. I found this chapter to be particularly interesting and relevant given the increasing severity of comorbid illnesses and complex surgical procedures, and the results of the recent POISE trial and other perioperative trials. This chapter will be a helpful guide for managing the complex surgical patient and for administering limited intensive care resources.
I must admit I had a desire for more information and wished this chapter was lengthier and more detailed. The chapters dealing specifically with anesthesia in relation to esophagectomy, vascular surgery, pelvic trauma, and the elderly are well written up-to-date reviews. The focus of the chapter specific to medication is on levosimendan for the treatment of acute decompensating congestive heart failure. The chapter regarding advances in critical care medicine covers the expanding role of echocardiography from the operating room to the intensive care unit. It includes a discussion by critical care outreach and medical emergency teams regarding the controversy within the current literature, and it incorporates a review of severe acute respiratory syndrome and pandemic planning. The final three chapters evaluate clinical performance, reduction of harm, and simulation to enhance training.

In summary, Recent Advances in Anaesthesia and Intensive Care encompasses a wide range of topics, from the pre-admission clinic, to the operating room, to the intensive care unit, to quality improvement. The book is directed more towards the anesthesiologist who is an intensivist rather than towards other medical specialties. Its focus is on preparing the critically ill patient for surgery, and it includes relevant sections for all persons involved with the critically ill patient, including medical emergency teams and those engaged in simulation. The chapters are short, easy to read, well researched, and well written. For a speedy and succinct read, this book would be an excellent addition to your library.

D. R. Townsend, MD ( $\square)$

University of Alberta, Edmonton, Canada

e-mail:drt1@ualberta.ca 